\title{
Comprehensive Characterization of Silicon Photomultipliers for Nuclear Security Applications
}

David L. Chichester, Marc Wonders, Marek Flaska

October 2017

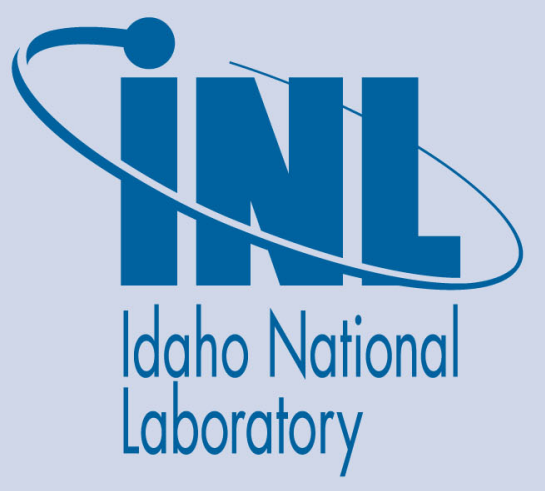

The INL is a U.S. Department of Energy National Laboratory operated by Battelle Energy Alliance 


\title{
Comprehensive Characterization of Silicon Photomultipliers for Nuclear Security Applications
}

\author{
David L. Chichester, Marc Wonders, Marek Flaska
}

October 2017

Idaho National Laboratory Idaho Falls, Idaho 83415

http://www.inl.gov

Prepared for the

U.S. Department of Energy

National Nuclear Security Administration

Under DOE Idaho Operations Office

Contract DE-AC07-05ID14517 


\title{
Comprehensive Characterization of Silicon Photomultipliers for Nuclear Security Applications
}

\author{
Marc A. Wonders, David L. Chichester, Senior Member, IEEE and Marek Flaska, Member, IEEE
}

\begin{abstract}
Scintillation detectors are an essential instrument in a variety of fields, serving as an effective means of detecting radiation for industrial, defense, medical, and basic-research applications. Light sensors are an integral part of all scintillation detectors and can affect detector performance as much as the scintillator itself. The photomultiplier tube (PMT) has been the standard light sensor for over half a century, but alternatives have been developed recently, and the silicon photomultiplier (SiPM) is perhaps the most promising of these new technologies. SiPMs can produce gains of the same order of magnitude as most PMTs and have shown reduced noise levels in the newest generations. Additionally, they posses benefits characteristic of solid-state devices such as insensitivity to magnetic fields, mechanical ruggedness, compactness, low operating voltages, and high quantum efficiencies. The timing properties of light sensors are an important characteristic for many applications, including nuclear security. For example, accurate timing is necessary for time of flight experiments, scatter cameras, and background suppression. This work aims to characterize the time resolution of many commercially available new-generation SiPMs, specifically in a time-coincidence setting. SiPMs have been acquired from five leading manufacturers possessing a variety of pixel and microcell sizes. Results are presented with an organic scintillator p-terphenyl and coincidence time resolution of less than 300 picoseconds is achieved. Comparison with PMTs shows that the PMT pair tested has a superior time resolution of 83 picoseconds.
\end{abstract}

\section{INTRODUCTION}

$\mathrm{S}_{\mathrm{d}}^{\mathrm{n}}$ ILICON photomultipliers (SiPMs) are one of the most recent $S_{\text {developments in the evolving line of solid-state light }}$ sensors. Depending on the manufacturer, they are sometimes called multi-pixel photon counters, Geiger-mode avalanche photodiodes, solid-state photomultipliers, etc., but all such devices will be referred to as SiPMs in this paper. As alluded to by their various names, SiPMs are essentially an array of many miniaturized avalanche photodiodes connected in parallel and operating in Geiger mode. Each avalanche photodiode is connected in series with a quenching resistor to form a microcell. Typical microcell sizes are tens of $\mu \mathrm{m}$ in dimension enabling thousands of microcells to be fit on pixels that are $\mathrm{mm}$ in dimension, the usual size of SiPMs. By operating in Geiger mode, each microcell basically operates as a binary switch that yields a gain similar to most

Manuscript submitted November 10, 2017. This material is based upon work supported by the US Department of Energy's National Nuclear Security Administration Office of International Nuclear Safeguards, from the Human Capital Development Subprogram.

M. A. Wonders and M. Flaska are with the Department of Mechanical and Nuclear Engineering, Pennsylvania State University, University Park, PA 16802-1412 (email: mfw5173@psu.edu,mflaska@psu.edu)

D. L. Chichester is with Idaho National Laboratory, Idaho Falls, ID 83415 (email: david.chichester@inl.gov) photomultiplier tubes (PMTs) when a photon is detected, and the large microcell number fill density allows for the output current to be proportional to the number of incident photons. Further, SiPMs require low operating voltages and possess high quantum efficiency, insensitivity to magnetic fields and mechanical shocks, and compact sizes. These characteristics make SiPMs effective light sensors for use in scintillation detectors.

Two additional characteristics of SiPMs also warrant consideration when assessing their suitability in specific applications: the noise and time profile of their voltage pulses. SiPMs traditionally have had higher noise levels than PMTs, though improvements in manufacturing and SiPM technology have reduced the noise in recent generations. SiPMs also generally have longer pulses, both in the rise time and overall time width of the pulse. These factors influence the timing resolution achievable in SiPM-based detectors as they can make effective time pickoff more difficult. Time resolution is important for detection systems in a variety of fields such as medical physics (positron-emission tomography), particle physics (particle identification), and nuclear security (scatter cameras, detector characterization, etc.). Consequently, a number of studies have attempted to determine the best time resolutions achievable with SiPMs [1]-[3]. Most of these use some sort of external amplifier with the SiPM and impressive time resolutions below 200 ps have been shown. However, further optimization of time resolution for SiPM based detectors may be possible. A comprehensive characterization of the state of the art in silicon photomultipliers is under way for multiple performance characteristics and a wide range of silicon photomultipliers. This paper reports on progress in the characterization of timing properties for many SiPMs and which SiPM characteristics most strongly influence time resolution.

\section{Materials AND Methods}

Twenty different SiPMs from five leading manufacturers have been acquired for characterization, but only fourteen are tested for these preliminary results [4]-[8]. The different types are shown in Fig. 1 and listed in Table I. To assess the time resolution of each SiPM type, two SiPMs of that type were set up on a breadboard with a ${ }^{22} \mathrm{Na}$ source placed in between. A 6-mm x 6-mm x 6-mm p-terphenyl crystal was used as the scintillator for both SiPMs in each pair, and the crystals are shown in Fig. 2. The crystals were wrapped with reflective Teflon tape and coupled to the SiPM with EJ-550 optical grease from Eljen Technology [9]. To shorten the rise time of SiPM pulses, a low-pass filter was placed on the bias supply line for each SiPM. The setup is shown in Fig. 3, and 
the waveforms from each SiPM are shown in Fig. 4. The measurements were conducted in a light-tight box.

TABLE I. SiLICON PHOTOMULTIPLIERS TESTED

\begin{tabular}{llll} 
Company & $\begin{array}{l}\text { Pixel } \\
\text { Size }(\mathrm{mm})\end{array}$ & $\begin{array}{l}\text { Microcell } \\
\text { Size }(\mu \mathrm{m})\end{array}$ & Series \\
\hline AdvanSiD & 4 & 40 & ASD-NUV \\
First Sensor & 4 & 40 & NUV \\
Hamamatsu & 3 & 25 & S13360 \\
Hamamatsu & 3 & 50 & S13360 \\
Hamamatsu & 3 & 75 & S13360 \\
Hamamatsu & 6 & 25 & S13360 \\
Hamamatsu & 6 & 50 & S13360 \\
Hamamatsu & 6 & 75 & S13360 \\
Ketek & 3 & 15 & WB \\
Ketek & 3 & 25 & WB \\
Ketek & 6 & 25 & EB \\
Ketek & 6 & 50 & EB \\
SensL & 3 & 35 & C \\
SensL & 6 & 35 & C \\
\hline
\end{tabular}

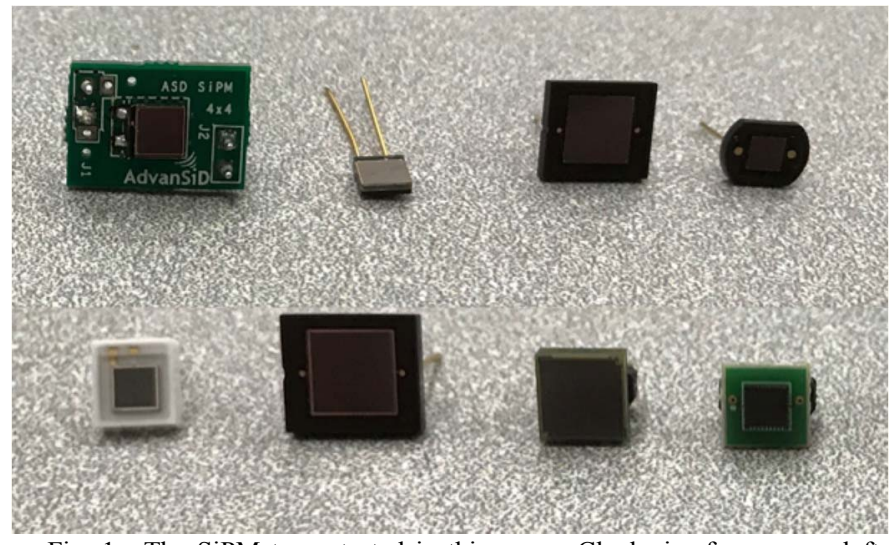

Fig. 1. The SiPM types tested in this paper. Clockwise from upper left: AdvanSiD 4 mm, First Sensor 4 mm, SensL 6 mm, SensL 3 mm, Ketek 3 mm, Ketek 6 mm, Hamamatsu 6 mm, Hamamatsu 3 mm.

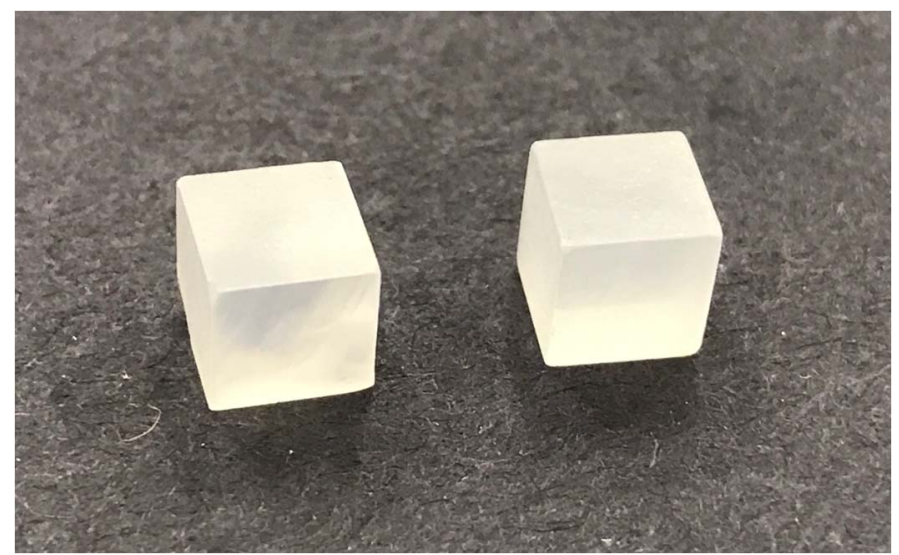

Fig. 2. Two 6-mm x 6-mm x 6-mm p-terphenyl crystals used in this study.

To record data, a CAEN DT5730 digitizer with 14-bit resolution, 500-MHz sampling rate, and $2-\mathrm{V}$ dynamic range was configured to only record events that occur in coincidence within a 24-ns window. Raw waveforms were collected and analyzed offline using the ROOT data analysis framework [10]. Time pickoff for each waveform was done using a constant fraction technique. A six-degree polynomial was fit to the rising edge of the pulse from the first sample below the chosen fraction to the second sample above it. A six-degree polynomial was chosen because it could smoothly and accurately model the portion of the rising edge while still being computationally fast in the ROOT framework. To determine the best fraction for each setup, the analysis code was automated to process the data using many different fractions between 4 and $20 \%$. An example pair of pulses with the fit is shown in Fig. 5. Pulses were required to have at least the pulse height of half the Compton edge for the 511-keV gamma rays, and a histogram was filled with the time difference of all events in a measurement. A Gaussian distribution was then fit to the distribution of events. An example histogram is presented in Fig. 6. The time resolution was taken to be the full-width half-maximum of the Gaussian fit. Previous work in [11] revealed the optimal overvoltage for pulse shape discrimination for each SiPM, and the overvoltage for each SiPM was set to the optimal pulse shape discrimination overvoltage or slightly higher.
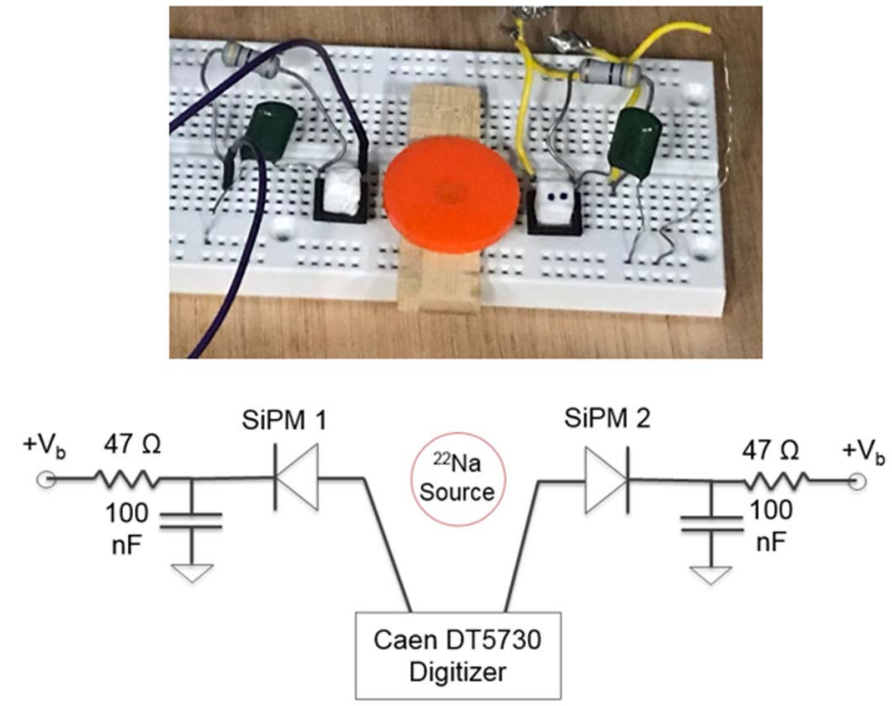

Fig. 3. The setup used for coincidence measurements. A low pass filter comprised of a $47-\Omega$ resistor and a $100-\mathrm{nF}$ capacitor was placed on each bias line and the signal was read out directly to the digitizer.

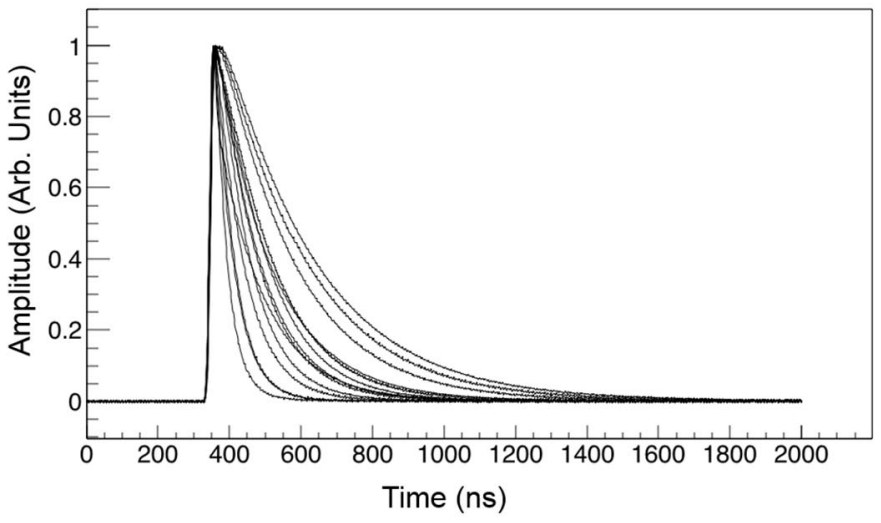

Fig. 4. Averaged waveforms of the SiPMs tested. The pulse widths are seen to span a wide range of lengths. 


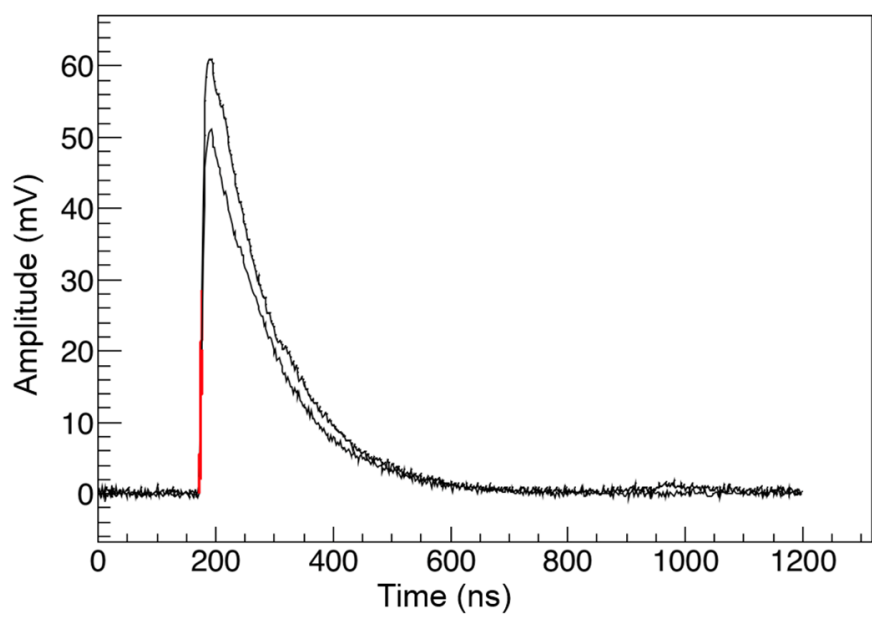

Fig. 5. Example pair of coincident pulses with fits to the rising edge shown in red.

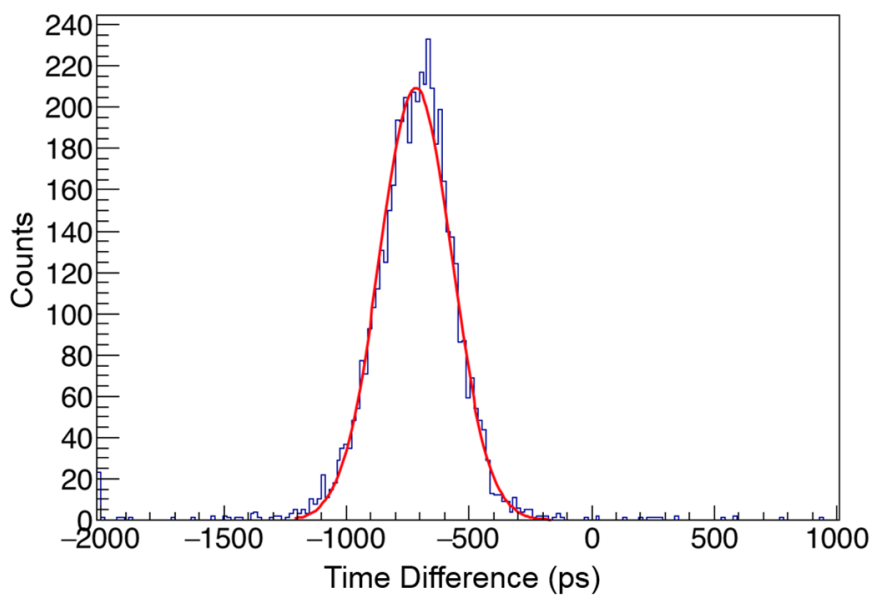

Fig. 6. Example distribution of time differences for a SiPM pair with a Gaussian fit to it in red.

\section{RESULTS}

The time resolution for all SiPMs tested is presented in Table II. Also shown is the rise time for each SiPM, where the rise time of a pulse is defined as the time difference between the peak sample and the last sample below $5 \%$ of the pulse height prior to the peak sample. The mean of a Gaussian fit to the distribution of many pulses was taken as the rise time for that SiPM.

In an attempt to determine what factors influence time resolution, a size figure was defined according to (1).

Size Figure $=$ Area of SiPM * Microcell Size

The relationship between the size figure and time resolution is shown in Fig. 7, and it is observed that in general as the size figure increases, the time resolution tends to improve. Because the crystal used has a 6-mm face, the area of the SiPM is proportional to its light collection efficiency, and as shown in [11], larger microcells generally correspond to higher signal to noise ratios. These factors help explain the observed trend in Fig. 7. The relationship between rise time and time resolution is also shown in Fig. 8, but no clear trend is observed.
TABle II. Silicon PhotomultiplieRs Tested

\begin{tabular}{lllll}
$\begin{array}{l}\text { Silicon } \\
\text { Photomultiplier }\end{array}$ & $\begin{array}{l}\text { Pixel Size } \\
(\mathrm{mm})\end{array}$ & $\begin{array}{l}\text { Microcell } \\
\text { Size }(\mu \mathrm{m})\end{array}$ & $\begin{array}{l}\text { Rise } \\
\text { Time } \\
(\mathrm{ns})\end{array}$ & $\begin{array}{l}\text { Full-Width } \\
\text { Half-Max } \\
(\mathrm{ps})\end{array}$ \\
\hline Hamamatsu 6075 & 6 & 75 & 17.8 & 293 \\
Hamamatsu 6050 & 6 & 50 & 18.1 & 288 \\
Hamamatsu 6025 & 6 & 25 & 18.6 & 409 \\
Ketek 6650 EB & 6 & 50 & 19.8 & 284 \\
Ketek 6625 EB & 6 & 25 & 19.1 & 444 \\
SensL 60035 & 6 & 35 & 19.4 & 335 \\
AdvanSiD 4040 & 4 & 40 & 17.3 & 423 \\
First Sensor 4040 & 4 & 40 & 17.5 & 442 \\
Hamamatsu 3075 & 3 & 75 & 8.0 & 466 \\
Hamamatsu 3050 & 3 & 50 & 10.3 & 405 \\
Hamamatsu 3025 & 3 & 25 & 11.5 & 501 \\
Ketek 3325 WB & 3 & 25 & 16.9 & 514 \\
Ketek 3315 WB & 3 & 15 & 17.0 & 603 \\
SensL 30035 & 3 & 35 & 17.5 & 475 \\
\hline
\end{tabular}

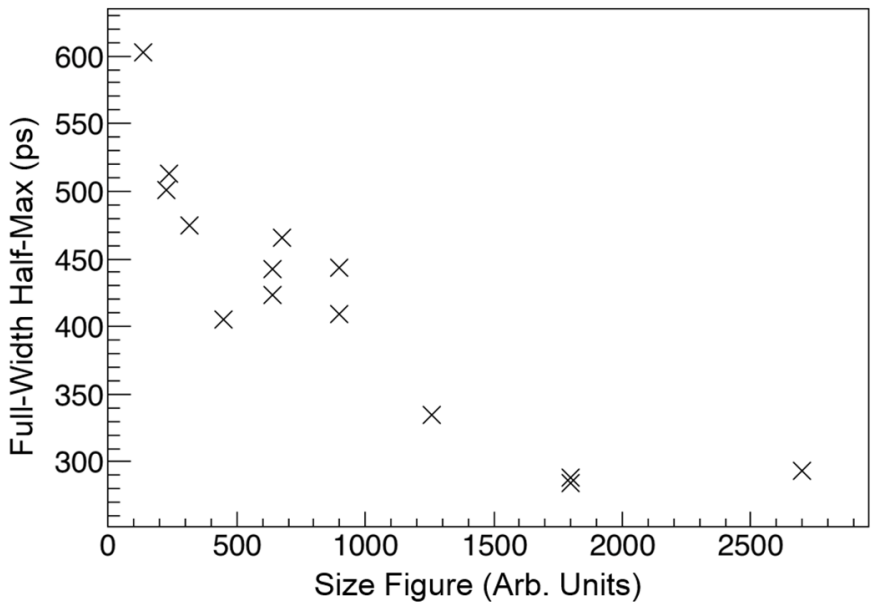

Fig. 7. Relationship between the size figure and time resolution for all SiPMs tested.

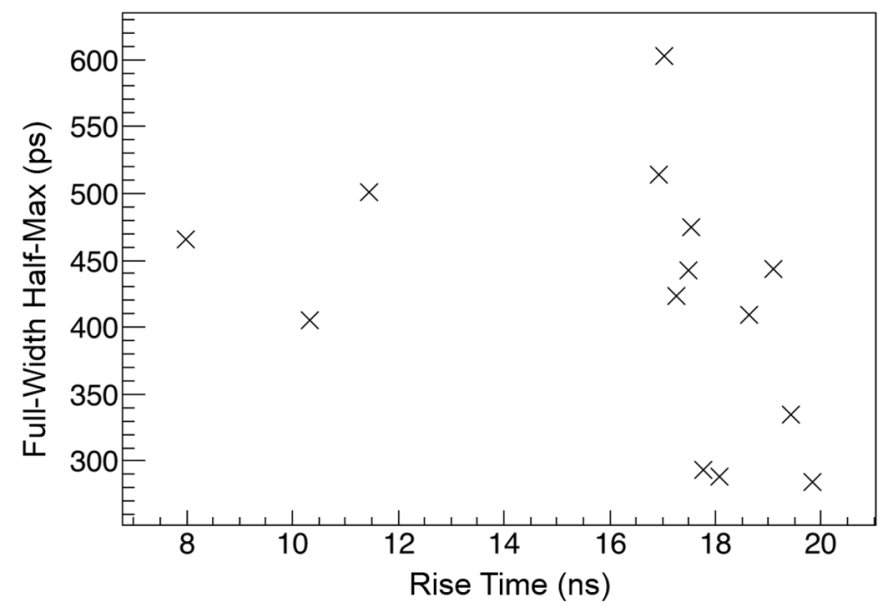

Fig. 8. Relationship between the rise time of SiPM pulses and time resolution for all SiPMs tested.

The time resolution for a pair of Hamamatsu H10580 PMT assemblies was also assessed with the same p-terphenyl crystals. The setup is shown in Fig. 9. The pulses produced by the PMTs are vastly different from those of most SiPMs 
tested. A comparison of waveforms for the PMT and the Ketek 6650 EB SiPM is shown in Fig. 10. While the Ketek 6650 EB SiPM showed the best time resolution, it should also be noted that it has one of the longest pulses. Both a larger microcell size and pixel size tend to produce longer pulses. The same method was used for time resolution determination except that the fit to the rising edge was simply a line fit to the sample above and below the chosen fraction because of the speed of the rising edge. The histogram of time differences for the PMT events is displayed in Fig. 11, and Table III compares the PMT pair with the Ketek 6650 EB pair.

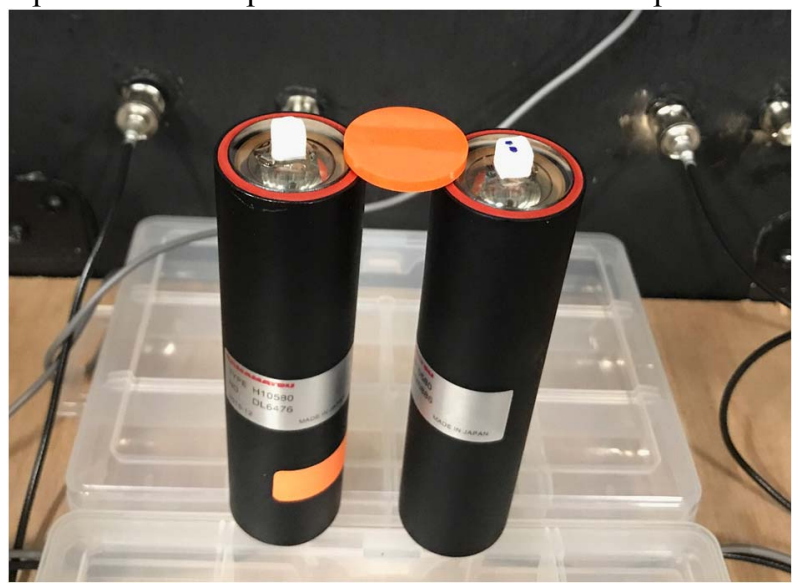

Fig. 9. Experimental setup for coincidence time resolution of a pair of Hamamatsu H10580 PMT assemblies.
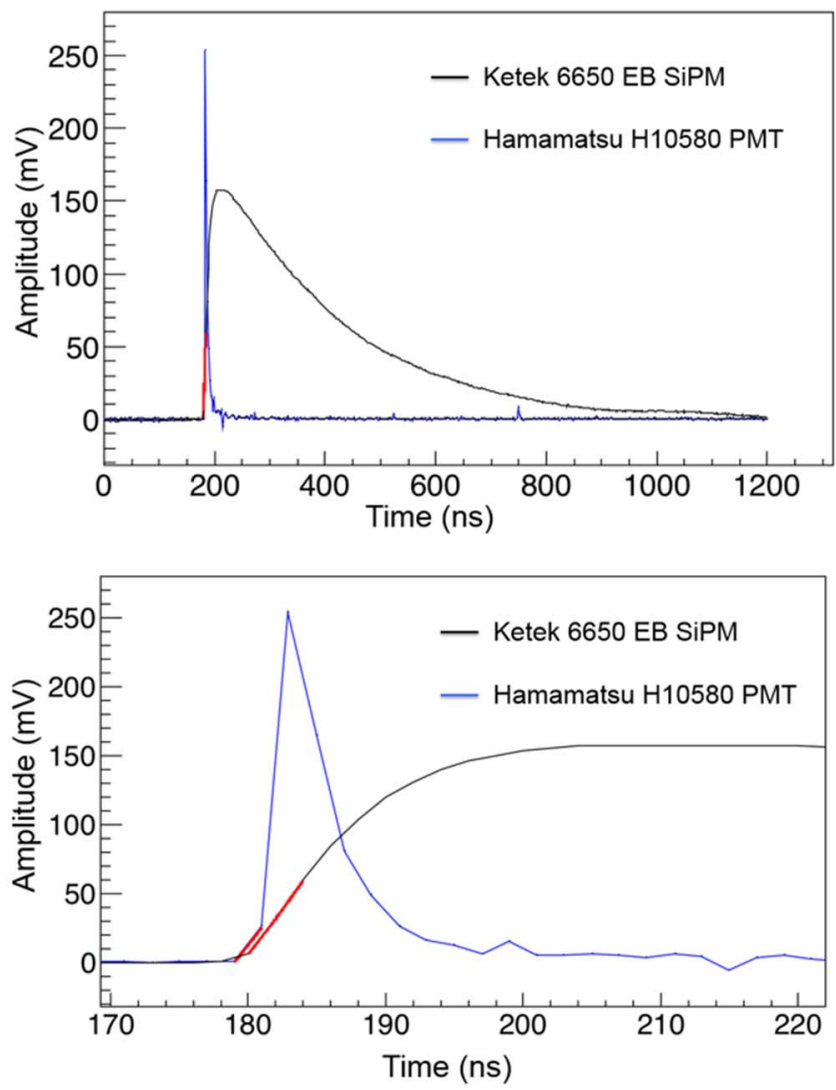

Fig. 10. Waveform comparison between pulses from a PMT and a SiPM. The SiPM chosen is the one that displayed the best time resolution and has longer pulses than most tested.

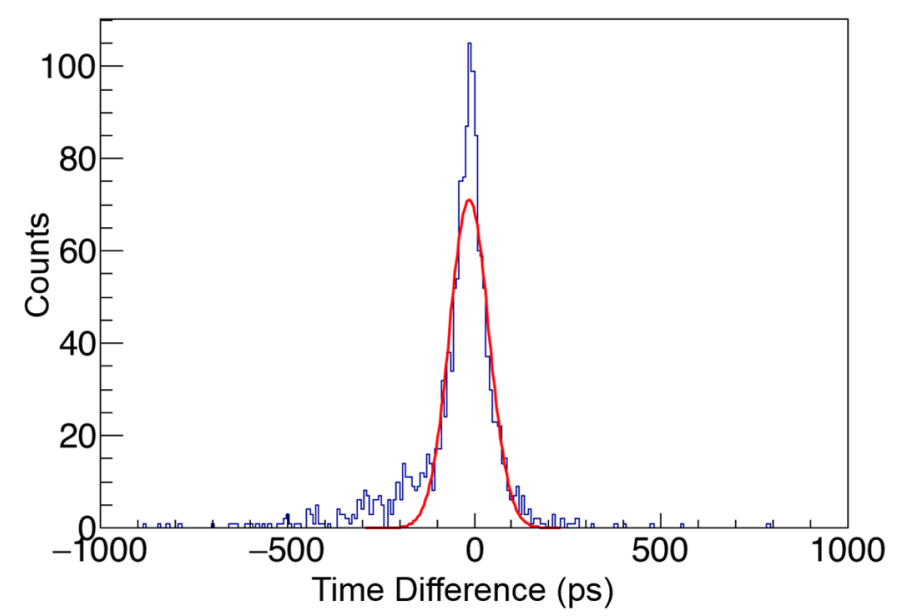

Fig. 11. Histogram of time differences in coincident events for the pair of Hamamatsu H10580 PMT assemblies. A Gaussian fit is shown in red.

TABLE III. PHOTOSENSOR COMPARISON

\begin{tabular}{lll} 
Photosensor & $\begin{array}{l}\text { Average Compton } \\
\text { Edge }(\mathrm{mV})\end{array}$ & $\begin{array}{l}\text { Full-Width } \\
\text { Half-Max }(\mathrm{ps})\end{array}$ \\
\hline Hamamatsu 10580 PMT & 443 & 83 \\
Ketek 6650 EB SiPM & 263 & 284 \\
\hline
\end{tabular}

\section{CONCLUSIONS}

Results for a variety of current-generation SiPMs shows effective time resolution on the order of hundreds of picoseconds, with all tested having a coincidence time resolution better than or equal to 603 picoseconds and three SiPMs having a coincidence time resolution less than 300 picoseconds. Nonetheless, the PMT pair showed an extremely impressive coincidence time resolution under 100 picoseconds, likely aided by larger and faster pulses than those of the SiPMs. The dominant characteristic affecting SiPM time resolution under this setup appears to be the product of light collection efficiency and gain, while the rise time itself does not appear to influence the time resolution. This indicates that the SiPM and digitizer noise likely plays a significant role in limiting effective time pickoff in the setup used.

\section{FUTURE WORK}

In addition to the results presented here, even more comprehensive testing is underway including testing of the other six SiPMs acquired. Each SiPM pair here is only tested at a single overvoltage, and a scan of time resolution as a function of overvoltage is necessary for a full characterization of SiPM time resolution. Other methods of time pickoff are also under investigation and multiple scintillators - organic and inorganic - are also being tested. Finally, characterization of time resolution as a function of temperature will also be performed.

\section{REFERENCES}

[1] M. V. Nemallapudi, S. Gundacker, P. Lecoq, and E. Auffray, "Single photon time resolution of state of the art SiPMs," Journal of Instrumentation, vol. 11, Oct. 2016. 
[2] S. Gundacker, et al., "A systematic study to optimize SiPM photodetectors for highest time resolution in PET," IEEE Trans. Nucl. Sci., vol. 59, no. 6, pp. 1798-1804, Oct. 2012.

[3] R. Vinke, et al., "Optimization of digital time pickoff methods for $\mathrm{LaBr}_{3}$-SiPM TOF-PET detectors," IEEE NSS Conference Record, pp. 2962-2968, 2009.

[4] AdvanSiD, Trento, Italy

[5] First Sensor, Berlin, Germany

[6] Hamamatsu, Shizuoka Prefecture, Japan,

[7] Ketek, Munich, Germany,

[8] SensL, Cork, Ireland

[9] Eljen Technology, Sweetwater, Texas, United States of America

[10] R. Brun and F. Rademakers, "ROOT - An object oriented data analysis framework," Nucl. Instr. and Meth. A., vol. 389, no. 1-2, pp. 81-86, Apr. 1997.

[11] M. A. Wonders, D. L. Chichester, M. Flaska, "Assessment of Performance of New-Generation Silicon Photomultipliers for Simultaneous Neutron and Gamma Ray Detection," IEEE Trans. Nucl. Sci., submitted for publication. 